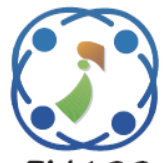

\title{
Short-Term Stock Market Fuzzy Trading System with Fuzzy Capital Management
}

\author{
Ahmed Tealab $^{1 *} \quad$ Hesham Hefny $^{1} \quad$ Amr Badr $^{1}$ \\ ${ }^{I}$ Computer Science Department, Institute of Statistical Studies and Research, Cairo University, Giza, Egypt \\ * Corresponding author's Email: a.tech.gouda@gmail.com
}

\begin{abstract}
Forecasting financial market indices became a necessary operation for investors' decisions in order to get the maximum return of investments. The complex behavior of the stock market requires development of forecasting systems. This work proposes a short-term stock fuzzy decision system using a novel trading strategy based on mixture of technical indicators. Fuzzy logic is applied for both trading rules definition and portfolio management. The selected stock market technical indicators for designing trading rules consist of commonly used indicators and new developed one. That is based on daily candlestick information produces short and long entry signals. The proposed system is tested using the Athens Stock Exchange data. The results are compared to classical non-fuzzy systems in addition to latest fuzzy approaches. The proposals performance produced less losses and better profits. The results demonstrate that the fuzzy logic is promising in portfolio management with steady upward profit and low losses. However, that deserves more study.
\end{abstract}

Keywords: Prediction, Fuzzy logic, Stock market, Short-term time series.

\section{Introduction}

Stock market has tight relation with countries economic. Finding the best time to buy or sell indices is the main challenge for experts and that requires prediction of market moves. Stock market indices behavior in general is quite noisy, nonlinear, and chaotic by nature [1]. It is not in random moves and can be classified as nonlinear time series with strong relation to previous data [2]. Different factors such as inflation reports, short term interest rate, political events, traders' expectations, and environmental factors are interpreted in stock prices moves causing market fluctuations which will make forecasting stock prices a difficult task. However, the evolution of technology and computer systems have affected the field of stock market forecasting in a way leads to develop financial forecasting and decision systems [3]. The use of intelligent systems such as neural networks [4], fuzzy logic [5] and genetic algorithms [6] in financial forecasting has several applications. Many technical indicators are being used in stock market prediction producing different results, their types include: Trend, Volume, Oscillators and Volatility indicators. Technical analysts and trading experts use one or more technical indicator in their market analysis and trading strategies.

This paper is organized as follows: Section 2 gives a background about related work. Technical analysis, soft computing and decision support systems are explained in Section 3. Section 4 describes Stock market prediction and money management model. Section 5 presents the experimental results. Finally, Section 6 concludes our work with a scope for future work.

\section{Background}

\subsection{Technical indicators and technical analysis}

Stock investor has to risk purchasing investments to get higher returns. Technical analysis is exploited as stock prices trends forecasting technique by studying historical indices' data. Thus, according to Efficient Market Hypothesis (EMH) 
studying specific technical indicators is enough to forecast prices moves and trends. Experts use combination of technical analysis techniques to forecast market moves by studying market history. They usually make subjective assessments to the data before developing their trading strategy; it is a difficult task and may have contradictory results [7]. Fuzzy models can address these issues.

Most of studies use common technical indicators derived from Moving Average (MA) such as Relative Strength Index (RSI) and Stochastic Oscillator (SO). Successful stock market forecasting strategies will be adapted by market and won't be successful any more. However, using the previous studied trading rules may give different profitable results for future periods and continues success is not guaranteed [8]. Studies found that technical trading strategies based MA rules won't yield profit with time [9].

Distinguishable characteristics of the indicators determine their profitability according to the market conditions. Furthermore, most of the common indicators are calculated for the previous sessions close price only, while there are other session data can be considered such as open, high and low price values. Therefore, it is obvious that there is a need for new technical indicators choices that are calculated based on full session data for better trading decisions based information. Since each technical indicator is developed for specific purpose, so combining different indicators may negate each other resulting in non-desired performance [10].

\subsection{Decision support systems}

Decision support systems (DSS) have promoted firstly from simple model-oriented systems to advanced multi-function entities [11]. There is a need to find the suitable DSS framework for each industry [12]. Thus, the financial industry has less work to be done, in particular, in the stock market filed. DSS were not advanced enough to fulfill financial decision takers needs [13]. However, some studies proposed intelligent decision systems based on studying the historical and current market data [14]. Before the exploration of soft computing, most of studies were focusing on time series analysis such as ARIMA, ARCH, and GARCH [15]. However, developing various computational intelligence forms such as neural network, fuzzy logic and genetic algorithm to predict stock market prices is needed.

\subsection{Soft computing}

Soft computing (SC) is the use of inexact and

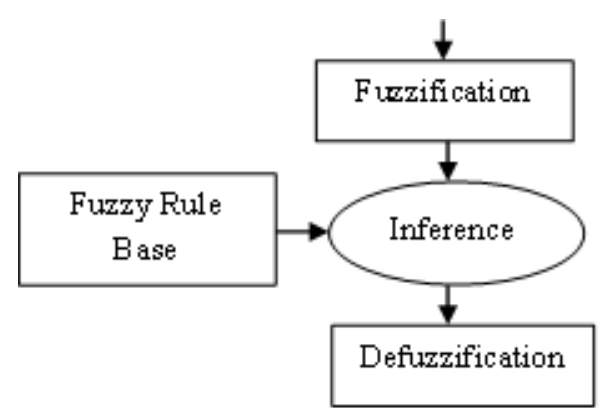

Figure 1. Fuzzy decision system

uncertain solutions for computationally hard tasks where there is no known algorithm to achieve tractability for low cost. Fuzzy logic, artificial neural network, particle swarm optimization and genetic algorithms amongst others are the principal constituents of Soft Computing. Fuzzy logic is defined by Zadeh [16] as a set of mathematical principles based on degrees of membership rather than classical binary logic that is used for knowledge representation Hybrid neuro-fuzzy model for stock market prediction [17], recursive possibilistic fuzzy modeling [18] in addition to the impact of sentiments from tweets as well as RSS news feeds using neural network [19] are successful applications in the real world. These studies resulted in significant improve in stock market prediction but without smart fuzzy management methodology.

Thus, it is apparent that a need of an intelligent system that can precisely and reliably perform prediction with portfolio management is important [20].

\section{Proposed systems}

The proposed system is identified through this section to explain its intelligent components. Three decision systems are considered, the first one has classical strategy. The other two are fuzzy systems, one of them has only fuzzy rules and the other has fuzzy rules and fuzzy capital management. Each system has one or more trading strategy. The trading strategy used in the proposed systems based on three indicators, two of them are known technical indicators and novel developed one.

The process of the fuzzy systems starts by fuzzifying the received input values them execute set of predefined IF-THEN-type rules when meet the reasoning conditions. The system major phases are (Fig. 1):

A) System architecture and operations definitions:

In this phase the whole system architecture and fuzzy system communication with the other system components are defined. 
B) Fuzzification: The process of transforming input values into fuzzy sets with assigned names and membership functions.

C) STFIS rule Base: It is a set of different STFIS rules, each has an "if - then" structure. "If" part specifies values of technical indicators and the "then" part specifies a rating given these values.

D) Defuzzification: In this phase the fuzzy output signal is transformed into a single number from predefined range of values and it represents the final system decision.

\subsection{Technical indicators}

The technical analysis concerns with the price movements in the market. Technical analysts attempt to use tools such as technical indicators, oscillators and charts to recognize patterns which can estimate future movement. In this study, a set of three technical indicators are used, the first one is the Stochastic Oscillator (SO). SO is momentum indicator; its value oscillates between 0 and 100 which represents the price of a security relative to the high/low range over a specific period of time. Its lines divergences and centerline crossovers can be used as indicator for overbought or oversold statuses. The calculations that draw stochastic oscillator lines are as following:

$$
\begin{aligned}
& \mathrm{K}=\frac{\left(C_{t}-L_{n}\right)}{\left(H_{n}-L_{n}\right)} \times 100 \\
& \mathrm{D}_{\text {fast }}=\operatorname{SMA}(3, \mathrm{~K}) \\
& \mathrm{D}_{\text {slow }}=\operatorname{SMA}(3, \mathrm{D})
\end{aligned}
$$

where $C_{t}$ is current price, $L_{n}$ and $H_{n}$ are the lowest and the highest close prices in last $n$ sessions respectively. $\operatorname{SMA}(n, \mathrm{x})$ is the simple moving average of variable $\mathrm{x}$ for $n$ sessions.

The recommended number of sessions by experts is $n=14$ which is the value used in this research. SO values above 80 reflect overbought conditions; on the opposite side; values under 20 are interpreted as indication of oversold conditions. The central line with value 50 represents neutral market momentum. In terms of market analysis and trading signals, SO that moves under 20 up to above 20 level is considered as bullish signal, while SO moves below 80 level coming from above 80 is viewed as bearish signal.

The second used indicator in this study is ADX (Average Directional Movement Index). It measures the strength of a trend of a financial time series. Its value based on DI+ and DI- which are the positive and negative directional indicators respectively. The indicator value is calculated from the following formulas:

$$
\begin{aligned}
& A D X=\frac{D I_{+}-D I_{-}}{D I_{+}+D I_{-}} \\
& D I_{+}=\frac{\text { Smoothed } D M_{+}}{T R} \\
& D I_{-}=\frac{\text { Smoothed } D M_{-}}{T R} \\
& D M_{+}=H_{t}-H_{t-1} \text { or } 0 \text { if result }<0 \\
& D M_{-}=-\left(L_{t}-L_{t-1}\right) \text { or } 0 \text { if result }<0
\end{aligned}
$$

where $D M_{+}$is Plus Directional Movement and $D M_{-}$ is the Minus Directional Movement, they determine the strength of bullish and bearish moves respectively, TR is the True Range, $\mathrm{H}_{t}, \mathrm{~L}_{\mathrm{t}}$ current high and low where $\mathrm{H}_{\mathrm{t}-1}, \mathrm{~L}_{\mathrm{t}-1}$ are previous high and low respectively. TR is largest absolute value of difference between highest and lowest value in $n$ periods. The recommended number of sessions by experts is 14 which is the value used in this research. The ADX generates a signal that quantifies the strength of an underway trend. Its value ranges between 0 and 100; ADX values less than 20 refer to trend weakness while values above 40 indicate trend strength as a common interpretation that is accepted among technical analysts.

The third indicator is the MyPivots (MP). MP is the new proposed indicator to determine market entry signals. MP is a customization of the Camarilla Pivot Points (CPP) indicator which commonly used by daily traders. The CPP lines are expected to determine intraday support and resistance levels; suggesting market entry and exit signals. The previous trading day High, Low and Close values are the input data for Camarilla equations to compose the new intraday levels. This method relies on the principle that the market price, which is a type of time series, tending to revert back to its mean value. Additionally, it is a type of trend following indicator too. It is useful with high sensitivity of MA based indicators which sometimes results in some false market entries. However, CPP is more precise for high volume and volatile stocks. Simply, these data are used as input parameters for the CPP equations to generate the new intraday support and resistance levels as follows:

$$
\begin{aligned}
& R 6=\left(H_{d-1} / L_{d-1}\right) \times C_{d-1} \\
& R 5=R 4+(R 4-R 3) \times 1.168 \\
& R 4=C_{d-1}+\left(H_{d-1}-L_{d-1}\right) \times 1.1 / 2 \\
& R 3=C_{d-1}+\left(H_{d-1}-L_{d-1}\right) \times 1.1 / 4 \\
& R 2=C_{d-1}+\left(H_{d-1}-L_{d-1}\right) \times 1.1 / 6
\end{aligned}
$$




$$
\begin{aligned}
& R 1=C_{d-1}+\left(H_{d-1}-L_{d-1}\right) \times 1.1 / 12 \\
& P P=\left(H_{d-1}+L_{d-1}+C_{d-1}\right) / 3 \\
& S 1=C_{d-1}-\left(H_{d-1}-L_{d-1}\right) \times 1.1 / 12 \\
& S 2=C_{d-1}-\left(H_{d-1}-L_{d-1}\right) \times 1.1 / 6 \\
& S 3=C_{d-1}-\left(H_{d-1}-L_{d-1}\right) \times 1.1 / 4 \\
& S 5=S 4-(S 3-S 4) \times 1.168 \\
& S 6=C_{d-1}-\left(R 6-C_{d-1}\right)
\end{aligned}
$$

where $\mathrm{R}$ and $\mathrm{S}$ refers to resistance and support levels respectively; $C_{d-1}, H_{d-1}$ and $L_{d-1}$ are Close, High, Low prices of the previous day respectively, while $\mathrm{PP}$ is the mean value and called Pivot Point line.

The Camarilla based trading strategy is to issue short position if the market reached up the area between R3 and R4 aiming the market to reverse down to PP. On the opposite side is to issue long position if the market price reached down the area between S3 and S4, expecting the market to revert up to PP. Another trading way with Camarilla is to follow the trend. It is concerned with the price breakout up of $\mathrm{R} 4$ or down from $\mathrm{S} 4$ generating bullish or bearish trend respectively. That will suggest issuing long position for bullish market and short position for bearish market trend.

The MP signal is based on two conditions come from the change in the market strength and determined by $C P P$ values as follows:

$$
M P=\left\{\begin{array}{c}
\text { Long } \\
\text { if } S 3 \geq O_{t}>S 4 \\
\text { and } P P \leq\left(H_{d-2}+L_{d-2}\right) / 2 \\
\text { Short } \\
\text { if } R 4>O_{t} \geq R 3 \\
\text { and } P P \geq\left(H_{d-2}+L_{d-2}\right) / 2 \\
\text { No Entry } \\
\text { if other }
\end{array}\right.
$$

where $O_{t}$ is the open value in at time $t$. In case of LONG and SHORT signals, their strength will be estimated using the following formula:

$$
\begin{aligned}
& \text { strength }= \\
& Q-\frac{3 \times\left(O_{t}-O_{t-1}\right)}{\max _{\text {diff_Open }}}-\frac{10 \times P P}{\max _{-} \text {diff_PP }} \\
& -\frac{3 \times\left(\text { Range }_{t-1}-\text { Rang }_{t-2}\right)}{\max _{-} \text {diff_Range }}
\end{aligned}
$$

where and max_diff_Open, max_diff_Range, max_diff_PP are the difference between the minimum and maximum values of all Open, Rnage and $P P$ values respectively in all sessions from 1 to $t$. $Q$ is the strength factor which is determined according the signal type as follows:

$$
Q=\left\{\begin{array}{l}
75 \text { if } M P \text { is Long } \\
25 \text { if } M P \text { is Short } \\
50 \text { if } M P \text { is No Entry }
\end{array}\right.
$$

The strength of long increases as the value of $Q$ is lower than 25 and while the higher the value of $Q$ more than 75 the stronger the short signal.

\subsection{Entry rules}

Entering the market for long or short position is based on the output signal of the decision system. The output signal generated from the combination of the trading indicators results in Bullish or Bearish trend. In bullish market prices are expected to rise, while in bearish market prices are expected to drop. Therefore, the suggested positions type is determined based on the type of the output signal; long position for bullish market and short position for bearish trend.

In this paper, the results of the decision system modelling with fuzzy set rules and the classical (crisp) methods are examined. The fuzzification of the technical indicators gives the information to generate trading rules. The crisp system based on technical analysis only. Additionally, the performance of the proposed systems are compared to results obtained by the short-term fuzzy trading system (STFS) proposed by K. Chourmouziadi [15].

\subsubsection{Classical system trading rules}

The classical system trading strategy is based on set of technical indicators which have a range of frequencies to compose the trading rules. The inputs are positive integer values of the technical indicators and the outputs are numeric values (-1: Bearish, 1: Bullish or 0: No Entry). Specific combination of the three antecedents indicators inputs mar represents certain market trend signal. The classical system output is signal for entering or existing market signal without strength qualification. Bullish and Bearish rules are shown in Table 1.

\subsubsection{Fuzzy trading rules}

The output signals of the decision systems are in the form of linguistic terms such as Bullish or Bearish in absence of any type of preciseness.

The imprecise output results in uncertainty of traders' decision which can be tolerated by using fuzzy logic. The experts believe that the market behavior is interpreted in the trader's decision system. However, that may keep producing signals 
Table 1. Crisp system rules

\begin{tabular}{lccccccc}
\hline if & $30<\mathrm{OS}<85$ & $\&$ & $25<\mathrm{ADX}<55$ & $\&$ & $\mathrm{~S} 4 \leq \mathrm{O}_{\mathrm{t}}<\mathrm{S} 3$ & then & 1 \\
elseif & $25<\mathrm{OS}<85$ & $\&$ & $55<\mathrm{ADX}<100$ & $\&$ & $\mathrm{~S} 4 \leq \mathrm{O}_{\mathrm{t}}<\mathrm{S} 3$ & then & 1 \\
elseif & $0<\mathrm{OS}<25$ & $\&$ & $25<\mathrm{ADX}<55$ & $\&$ & $\mathrm{~S} 4 \leq \mathrm{O}_{\mathrm{t}}<\mathrm{S} 3$ & then & 1 \\
elseif & $0<\mathrm{OS}<25$ & $\&$ & $0<\mathrm{ADX}<25$ & $\&$ & $\mathrm{~S} 4 \leq \mathrm{O}_{\mathrm{t}}<\mathrm{S} 3$ & then & 1 \\
elseif & $0<\mathrm{OS}<25$ & $\&$ & $55<\mathrm{ADX}<100$ & $\&$ & $\mathrm{~S} 4 \leq \mathrm{O}_{\mathrm{t}}<\mathrm{S} 3$ & then & 1 \\
elseif & $0<\mathrm{OS}<25$ & $\&$ & $25<\mathrm{ADX}<55$ & $\&$ & $\mathrm{~S} 4 \leq \mathrm{O}_{\mathrm{t}}<\mathrm{S} 3$ & then & 1 \\
elseif & $85<\mathrm{OS}<100$ & $\&$ & $25<\mathrm{ADX}<55$ & $\&$ & $\mathrm{R} 3<\mathrm{O}_{\mathrm{t}} \leq \mathrm{R} 4$ & then & -1 \\
elseif & $85<\mathrm{OS}<100$ & $\&$ & $0<\mathrm{ADX}<55$ & $\&$ & $\mathrm{R} 3<\mathrm{O}_{\mathrm{t}} \leq \mathrm{R} 4$ & then & -1 \\
elseif & $85<\mathrm{OS}<100$ & $\&$ & $55<\mathrm{ADX}<100$ & $\&$ & $\mathrm{R} 3<\mathrm{O}_{\mathrm{t}} \leq \mathrm{R} 4$ & then & -1 \\
elseif & $35<\mathrm{OS}<85$ & $\&$ & $25<\mathrm{ADX}<55$ & $\&$ & $\mathrm{R} 3<\mathrm{O}_{\mathrm{t}} \leq \mathrm{R} 4$ & then & -1 \\
elseif & $35<\mathrm{OS}<85$ & $\&$ & $55<\mathrm{ADX}<100$ & $\&$ & $\mathrm{R} 3<\mathrm{O}_{\mathrm{t}} \leq \mathrm{R} 4$ & then & -1 \\
elseif & $85<\mathrm{OS}<100$ & $\&$ & $25<\mathrm{ADX}<55$ & $\&$ & $\mathrm{R} 3<\mathrm{O}_{\mathrm{t}} \leq \mathrm{R} 4$ & then & -1 \\
else & & & & & & & 0 \\
\hline
\end{tabular}

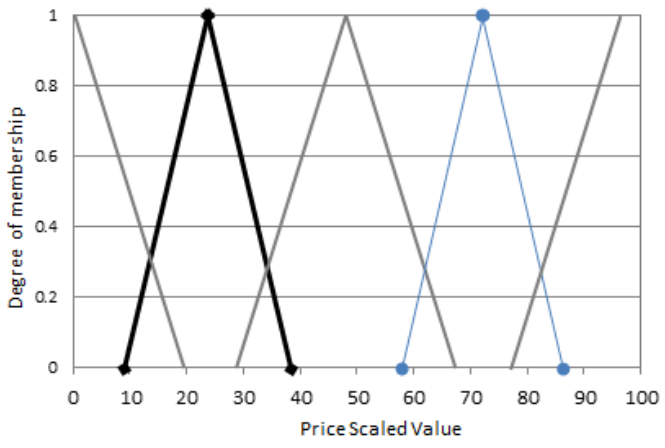

(a)

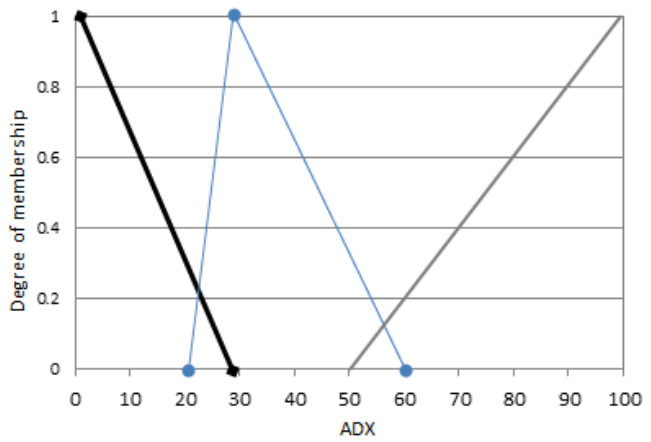

(c)

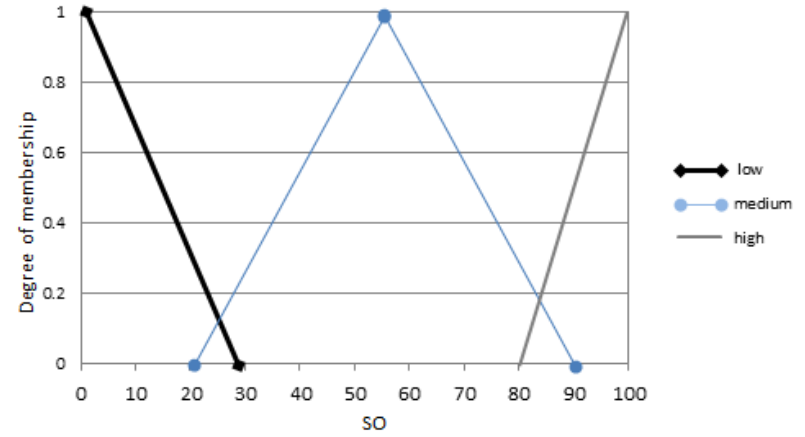

(b)

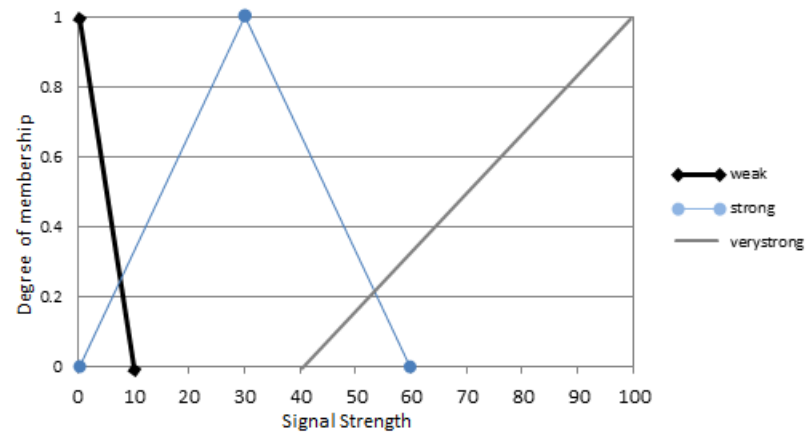

(d)

Figure. 2 Fuzzification: (a) MyPivots indicator, (b) SO indicator, (c) ADX indicator, and (d) output signal

based on imprecise inputs. ADX and SO indicators values between 0 and 100. In MP lines, by considering R6 is the top value and it is equal to 100 on the $0-100$ scale. Thus, the other MP lines on the $0-100$ can be found using the following formula:

$$
\text { nLine }=100 \times \text { Line } / \text { R6 }
$$

where nLine is the new MP line's value on the 0100 scale and Line is the real MP value.

Fig. 2 shows the fuzzification of the technical indicators and output conditions of the proposed system. The different input values of MP are classified to three fuzzy sets: LONG (LN) for long position, SHORT $(\mathrm{SH})$ for short position and WAIT (WT) for no position or no entry signals. The WAIT value represents a neutral case for market status between LONG and SHORT or extreme indicator values within its range. However, the traders believe that the market will reverse its position from these extreme locations (Fig. 2 (a)). The assigned fuzzy sets to each indicator are different from indicator to another one; it depends on the indicator output signal. The SO values vary between 0 and100 has assigned LOW (LO), MEDIUM (ME) and HIGH (HI) fuzzy sets for its outputs (Fig. 2 (b)). Similarly, the ADX indicators low, medium and high output values are assigned NOTREND (NO), ONTREND (ON) and RISKY (RIS) fuzzy sets respectively. The high ADX values considered RISKY cause it is almost come to the end of the trend (Fig. 2 (c)). 
Table 2. Fuzzy trading rules

\begin{tabular}{ccccc}
\hline OS & ADX & MP & Bullish & Bearish \\
\hline HI & ON & LN & WK & WK \\
HI & NO & LN & WK & WK \\
HI & RIS & LN & WK & WK \\
ME & ON & LN & VS & WK \\
ME & NO & LN & WK & WK \\
ME & RIS & LN & ST & WK \\
LO & ON & LN & VS & WK \\
LO & NO & LN & ST & WK \\
LO & RIS & LN & ST & WK \\
HI & ON & SR & WK & VS \\
HI & NO & SR & WK & ST \\
HI & RIS & SR & WK & ST \\
ME & ON & SR & WK & VS \\
ME & NO & SR & WK & WK \\
ME & RIS & SR & WK & ST \\
LO & ON & SR & WK & WK \\
LO & NO & SR & WK & WK \\
LO & RIS & SR & WK & WK \\
HI & ON & WT & WK & ST \\
HI & NO & WT & WK & WK \\
HI & RIS & WT & WK & WK \\
ME & ON & WT & WK & WK \\
ME & NO & WT & WK & WK \\
ME & RIS & WT & WK & WK \\
L & ON & W & ST & WK \\
L & NO & W & WK & WK \\
L & RIS & W & WK & WK \\
\hline & & & & \\
\hline
\end{tabular}

WEAK (WK), STRONG (ST) and VERYSTRONG (VS) are fuzzy sets with clear meanings; describe the bullish and bearish outputs (Fig. 2 (d)). For the generality and simplicity reasons, triangular membership functions represented all the fuzzy sets.

Table 2 contains defined set of possible indicators combinations that follow the behavior of the trading experts in formulating their trading rules. Consequently, the fuzzy sets of output signals (bullish and bearish) strength are (WEAK, STRONG and VERYSTRONG). That is interpreted in the system entry action to the market with different recommendation based on the strength level. The system output signals' strengths are STRONG (ST), VERYSTRONG (VS) and WEAK (WK) for both the Bullish and Bearish signals. The used fuzzy system is Mamdani [17] with maximum/minimum based operators. The centroid method is used in the defuzzification phase.

\subsection{Exit conditions}

The unpredictability of the after trades market moves might raise greed or fear emotional states. Thus, a method that defines market exit conditions and avoiding instant monitoring to market is required for any trading system. The setup of stop threshold conditions (stop-loss and stop-profit) is a protective step for the established positions to limit their maximum losses and revenues. Managing both losses and profits will ensure prudential revenue from unexpected extreme market moves or turns. This step will be used to calculate the amount of money to invest.

The volatility of the market at time of issuing a position will determine the (stop-loss and stopprofit) thresholds. However, the ATR (Average True Range) is used as volatility estimator. ATR is found for $q$ session which is equal to 14 as used with SO and ADX indicators. The ATR is defined as follows:

$$
\begin{gathered}
T R=\operatorname{Max}\left(M_{t}-m_{t}, M_{t}-c_{t-1}, m_{t}\right. \\
\left.-c_{t-1}\right)
\end{gathered}
$$

where $M_{t}, m_{t}$ represent the highest, lowest price values in a session $t$ respectilvey, while $c_{t-1}$ is close value of the previous session. In the proposed system the exit conditions are calculated and assigned at the position open time. For bullish market case, the stop-loss is the position open price minus twice the ATR value. To have balanced and conservative strategy with Risk/Reward ration 1/1, the stop-profit will also be the position open price plus twice the ATR value and vice versa for bearish market.

\section{Money management}

In the proposed system the output of the fuzzy trading rules is the input of the fuzzy capital management system. The fuzzy trading system is responsible to decide the amount of money to invest with a simple risk management strategy. Vince [21] proposed the optimal-F that provides a method to calculate the required amount of the investor's portfolio to achieve a geometric profits growth. Its way to evaluate exactly the money to be used in each trade is a capital percentage that is directly proportional to the previous winning trades. A customization has been done to the optimal-F that its input is replaced by the fuzzy output generated by the proposed intelligent system.

One approach here is the "fixed fractional trading" strategy, where an investor wants to risk a fixed percentage of his current capital for all trades and investments. The past investments yields affect the following capital available for investment. To maximize the Terminal Wealth Relative (TWR) for a specific trading period we have to calculate the optimal-F to find required the fraction (f) for that. Each trading operation $i$ has a return factor $H_{P R}(f)$ which is a function of the operation result return $_{i}$ and worst trade (drawdown) in the 
specified trading period. The following equations calculate the TWR:

$$
\begin{gathered}
T W R(f)=\prod_{i=1}^{2} H P R_{i}(f) \\
\operatorname{HPR}_{i}(f)=1+\frac{f .\left(\text { return }_{i}\right)}{\text { drawdown }}
\end{gathered}
$$

Therefore, the relative relation between the possible risk and the expected reward (Risk/Reward) for each trade means that targeting high profitable trades will require high optimal value of $f$ which is practically rare. A practical value of $f$ can be considered as percentage of optimal-F. In the proposed system, partial-F is introduced as $12 \%$ of the found optimal$\mathrm{F}$ as the possible applied $f$.

Fig. 3 demonstrates the Risk/Reward relation curve or the expected gain against the risk assumed. In the Gaussian curve it is clear that the highest point of the curve which represents the maximum expected profit (reward) is at the optimal-F. Therewith, it is possible to observe that with less risk, less profit is expected. Partial-F is located on the left side of the curve at $12 \%$ of the optimal-F. The fuzzy trading system determines the range amount of capital available for investment; it will be estimated as:

$$
\begin{aligned}
& \text { custom }_{f} \\
& =\text { partial }_{f} \\
& +\frac{\left(\text { signal }_{-} \text {min_signal }\right)\left(\text { optimal }_{f}-\text { partial }_{f}\right)}{\text { sginal }_{r} \times \text { optimal }_{f}}
\end{aligned}
$$

where signal is the system output (Bullish or Bearish); min_sginal is the minimum signal value to trigger a trade (its value is 15); optimal ${ }_{\mathrm{f}}$ and partial $_{f}$ are optimal-F and partial-F respectively;

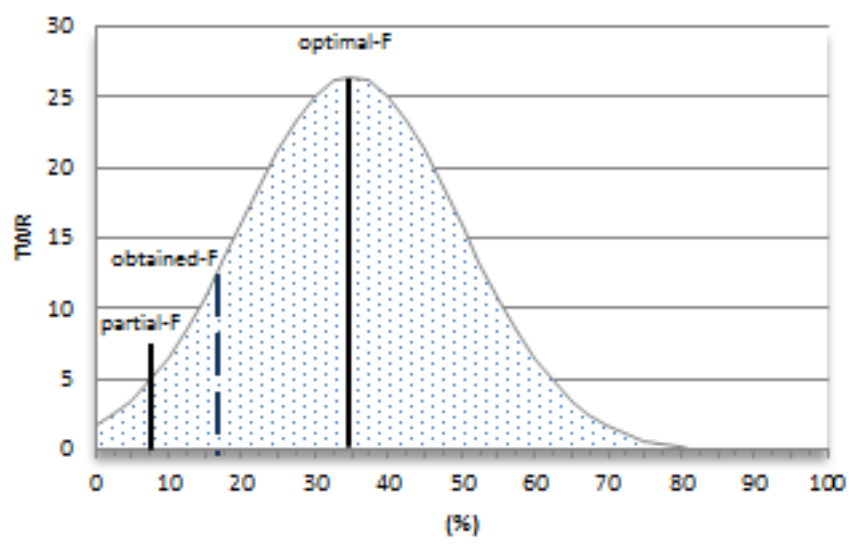

Figure 3. optimal-F, obtained-F and partial-F demonstration sginal $_{r}$ is difference between minimum and maximum of signals values. After obtaining the optimal-F, the next step is to calculate the required number of shares using the following formula:

$$
\# \text { shares }=\text { partial }_{f}+\frac{\text { equity }}{\text { stoploss }}
$$

where equity is the amount of money available for investment and stoploss is the market exit condition value. The above formula (18) output is estimation to the number of shares required for purchase without consideration of the share price. In case that the available money for investment isn't enough to cover the required number of shares, the maximum available number is used. But in the classical system it uses trading rules with all available capital for investment in all assigned positions.

\section{Experimental setup}

In this research the Metatrader Trader software (MT4) is used to implement the three trading systems and to compare their performance for the same database. To test these systems well, the criteria of selecting the validation market indices should include diversity of trends through the validation period.

The results obtained by K. Chourmouziadis [14] fuzzy system are used as our benchmark; they showed better performance than the B\&H strategy. Chourmouziadis [14] proposed a short-term fuzzy trading system (STFS) with technical based trading strategy and portfolio management; its fuzzy trading strategy uses set of appropriate technical indicators. Wherefore, general index of the Greek (Athens) stock market (ASE) is the validation data used by STFS [14]. Therefore, the proposed system in this research will be tested with the same dataset too without consideration of the transaction cost. Chourmouziadis [14] examined his system separately for bull and bear market periods between 15 November 1996 and 5 June 2012 covering different world and local economic events and changes. The ASE demonstrated different behavior through this period; it shows relatively long periods of bearish market before turning to a bullish trend then back to bearish again. It will be good test for the systems profitability in different situations.

The three decision systems (Fuzzy RC, Fuzzy R, and Classical) are distinguishable in their using to the fuzzy logic. Fuzzy RC: is the first system; it is a fuzzy decision system where fuzzy logic is applied 
Table 3. Systems performance: Bull markets

\begin{tabular}{|c|c|c|c|c|c|c|c|c|c|}
\hline \multicolumn{2}{|c|}{ Bull Markets, ASE } & \multicolumn{2}{c|}{ STFS Profit } & \multicolumn{2}{c|}{ Classic Profit } & \multicolumn{2}{c|}{ Fuzzy R Profit } & \multicolumn{2}{|c|}{ Fuzzy RC Profit } \\
\hline From & To & EUR & $\%$ & EUR & $\%$ & EUR & $\%$ & EUR & $\%$ \\
\hline $15-11-1996$ & $17-09-1999$ & 63630 & 536.3 & 58167 & 481.67 & 60324 & 503.24 & 77863 & 678.63 \\
\hline $01-04-2003$ & $09-03-2009$ & 27364 & 173.6 & 23816 & 138.16 & 25482 & 154.82 & 30144 & 201.44 \\
\hline $09-03-2009$ & $14-10-2009$ & 15027 & 50.2 & 12812 & 28.12 & 15631 & 56.31 & 16903 & 69.03 \\
\hline
\end{tabular}

Table 4. Systems performance: Bear markets

\begin{tabular}{|c|c|c|c|c|c|c|c|c|c|}
\hline \multicolumn{2}{|c|}{ Bear Markets, ASE } & \multicolumn{2}{c|}{ STFS Profit } & \multicolumn{2}{c|}{ Classic Profit } & Fuzzy R Profit & \multicolumn{2}{c|}{ Fuzzy RC Profit } \\
\hline From & To & EUR & $\%$ & EUR & $\%$ & EUR & $\%$ & EUR & $\%$ \\
\hline $20-09-1999$ & $31-03-2003$ & 9595 & -4.05 & 6686 & -33.14 & 9047 & -9.53 & 9802 & -1.98 \\
\hline $01-11-2007$ & $09-03-2009$ & 7383 & -26.17 & 7836 & -21.64 & 6995 & -30.05 & 7850 & -21.50 \\
\hline $15-10-2009$ & $05-06-2012$ & 4833 & -51.67 & 3456 & -65.44 & 3888 & -61.12 & 5079 & -49.21 \\
\hline
\end{tabular}

Table 5. Proposed systems profits and drawdown

\begin{tabular}{|c|c|c|c|c|c|c|c|}
\hline \multicolumn{2}{|c|}{ Market Periods } & \multicolumn{2}{c|}{ Classic } & \multicolumn{2}{c|}{ Fuzzy R } & \multicolumn{2}{c|}{ Fuzzy RC } \\
\hline FROM & TO & Profit \% & DD \% & Profit \% & DD \% & Profit \% & DD \% \\
\hline $15-11-1996$ & $17-09-1999$ & 481.67 & -29.08 & 503.24 & -25.30 & 678.63 & -27.10 \\
\hline $20-09-1999$ & $20-09-1999$ & -33.14 & -37.25 & -9.53 & -38.01 & -1.98 & -30.92 \\
\hline $01-04-2003$ & $01-04-2003$ & 138.16 & -24.71 & 154.82 & -20.41 & 201.44 & -14.06 \\
\hline $01-11-2007$ & $09-03-2009$ & -21.64 & -29.52 & -30.05 & -26.81 & -21.50 & -18.32 \\
\hline $09-03-2009$ & $09-03-2009$ & 28.12 & -22.17 & 56.31 & -18.24 & 69.03 & -11.51 \\
\hline $15-10-2009$ & $05-06-2012$ & -65.44 & -23.36 & -61.12 & -19.75 & -49.21 & -10.67 \\
\hline
\end{tabular}

on the information part for both the rules and indicators and on the estimation of the capital to invest using Eqs. (17) and (18). Fuzzy R: is the second system; it is also a fuzzy intelligent decision system but fuzzy logic is used only for the information part including the rules and technical indicators.

Although, the estimation of the capital to invest is done using the Partial-F with value equal to $12 \%$ of optimal-F. The third system is a classical trading system in absence of fuzzy logic in any of its parts. A drawdown (DD) during a specific recorded period is a measure in percent of the decline happens in an investment from peak-to-trough for that period. Trading systems are run over the validation period for the selected index (ASE), slippage adjusted and profits are calculated on the basis of a notional $€ 10,000$ (EUR) of the trading capital. Furthermore, the largest drawdown is calculated. Drawdown and trading profit for each trading period are presented in Table 5. The Fuzzy RC showed better performance than the Fuzzy R and Classical systems. The total net profit achieved by the Fuzzy RC system in bull markets and the losses in bear markets are the best in comparison to the other systems (Tables 3 and 4). The profit factor, the average profit over average loss ratio, performed by Fuzzy RC system is greater than 1, which resulted in a profitable trading system for the whole validation period. Figure (6) shows the performance impact of the three trading systems and SFTS [14] on the investment portfolio.
The final results indicate that the Fuzzy RC produced the best performance with total net profit $€ 145045.26$ (1450.5\%). Fuzzy $\mathrm{R}$ ended the validation period with total net profit $€ 59119.25$ $(491.2 \%)$ which is better than classical results with final profit $€ 22136.3$ (221.3\%). The STFS [14] performance is better than the Fuzzy R and classical systems but less than the Fuzzy RC system and that might be of the STFS [14] fuzzy capital management. Therewith, the Fuzzy RC showed better results in all trading periods than the STFS [14] including bear and bull markets. Therefore the importance of a trading system with those properties resulted in high trading operation (Figs. 4 and 5).

The proposed systems are examined for the associated maximum drawdown (DD) which is quite high for each system. In the classical trading system fewer trades are triggered which result in less profit and higher DD. But effect of high DD in the Fuzzy $\mathrm{RC}$ trading systems is compromised and over challenged with much higher profitable trades. The cumulative performance for ASE index alleviates the DD effect when the negative trades are not occurred in different period of profitable trades (Table 5). The results proved that the Fuzzy RC trading system is superior in comparison to the other two systems. It can be considered a very good trading system as it obtains higher profits and less risk. Nevertheless the behavior of the Fuzzy $\mathrm{R}$ is slightly better than the classic technique indicating that the combination of both the fuzzy indicators and 


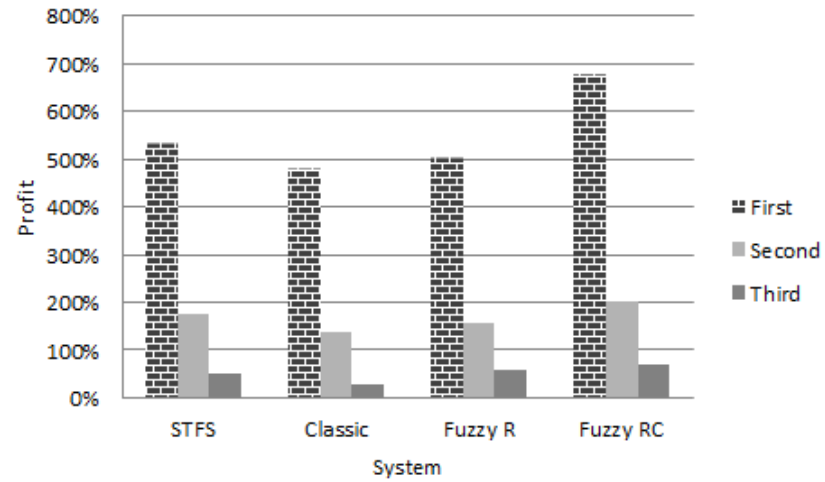

Figure. 4 Systems profit in bull marekts periods

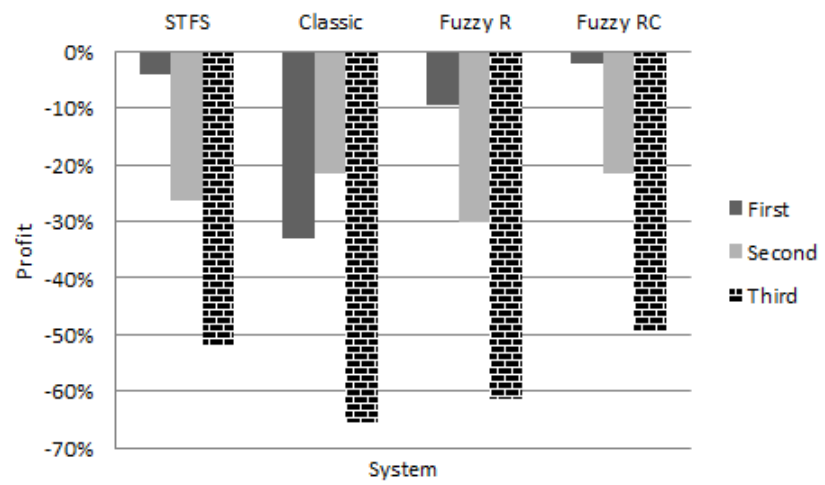

Figure. 5 Systems profit in bear marekts periods

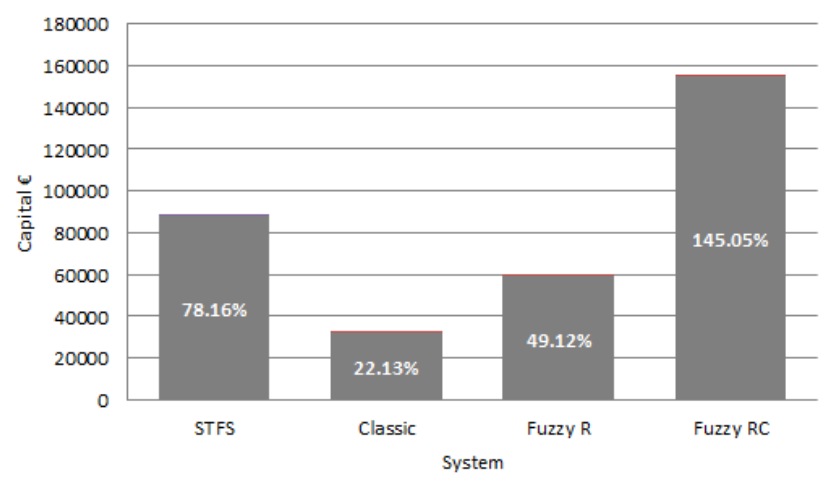

Figure. 6 Systems toal profit $\%$ for the whole validation period

the fuzzy capital management has a significant impact on the Fuzzy RC system performance.

With its intelligent decision, the Fuzzy RC trading system behaves well when classical system loses money avoiding high drawdown when the market goes down. Fuzzy RC performance is smooth and steady upward during the whole validation period. These features are quite desirable. The system produces steady profitability and robustness under different market conditions.

More attention should be paid to the use of fuzzy logic in trading systems not only for the indicators and rules but also for capital management. These experiments evidence maybe is not enough.

\section{Conclusions}

Fuzzy logic, by its nature, is a suitable technique to deal with uncertainty, impreciseness and vagueness problems; hence trading systems too. In this research, a short-term fuzzy decision system with fuzzy technical indicators and fuzzy trading rules in addition to fuzzy capital management is proposed. The system trading strategy is based on a set of known technical indicator and new developed one. The role of the trading system is to forecast market moves and to give advice for issuing positions with exit conditions. Additionally it gives recommendations on the amount of portfolio to invest. Therefore it is an important part of the fuzzy decision system as most of the proposed fuzzy decision systems in the literature don't include fuzzy capital management. Although, there are some articles proposed capital management from a fuzzy perspective [20, 22]. The validation of the trading systems has been done for general index of the Greek (Athens) stock market (ASE) over a period of fifteen years. The proposed system showed substantial performance with overall robust profit and low drawdown. The achieved results considered as evidence to encourage using fuzzy decision systems with fuzzy capital management. It is a promising techniques and merits further investigation. Regarding this, the proposed trading system requires more testing cases to further explore the system properties. The future work should include expansion of the experimental setup to cover independent assets of different sectors over other time periods. Furthermore it should belong with different real portfolios.

One of the advantages of trading tools based on fuzzy logic is the flexibility of controlling the decisions by the operator. Wherever, future research should concentrate on bringing this additional flexibility. That can be done by fuzzifying other classic trading strategies using the proposed ideas in this research. This would give us the chance to validate more the fitness of the proposed technique here. Nevertheless; an optimization to the technical indicators parameters can be done using techniques such as genetic algorithm.

Finally, the use of fuzzy logic can be extended to implement better risk control mechanism. That may help in determining the exit conditions with lower drawdown.

\section{References}

[1] R. Balvers, Y. Wu, and E. Gilliland, "Mean reversion across national stock markets and 
parametric contrarian investment strategies", The Journal of Finance, Vol. 55, No. 2, pp. 745-772, 2000.

[2] A. Rahman and S Sasdi, "Random walk and breaking trend in financial series: An econometric critique of unit root tests", Review of Financial Economics, Vol. 17, No. 3, pp. 204-212, 2008.

[3] W. Chiang, D. Enke, T. Wu, and R. Wang, "An adaptive stock index trading decision support system", Expert Systems with Applications, Vol. 59, No. 15, pp. 195-207, 2016.

[4] G. Armano, M. Marchesi, and A. Murru, "A hybrid genetic-neural architecture for stock indexes forecasting", Information Sciences, Vol. 170, pp. 3-33, 2005.

[5] P. Chang, C. Fan, and J. Lin, "Trend discovery in financial time series data using a case based fuzzy decision tree", Expert Systems with Applications, Vol. 59, No. 15, pp. 195-207, 2016.

[6] A. Tealab, A. Badr, and M. Fakhr, "Automatic FOREX Market Trading based on Technical Analysis", Computing and Information Systems Journal, Vol. 15, No. 1, 2012.

[7] G. Panda, G. Sahoo, and R. Majhi, "Development and performance evaluation of FLANN based model for forecasting of stock markets", Expert Systems with Applications, Vol. 36, No. 3, pp. 6800-6808, 2009.

[8] B. Malkiel, "Reflections on the Efficient Market Hypothesis: 30 Years Later", Financial Review, Vol. 40, pp. 1-9, 2005.

[9] C. Park and S. Irwin, "The Profitability of Technical Trading Rules in Us Futures Markets: A Data Snooping Free Test", http://dx.doi.org/10.2139/ssrn.722264, 2005.

[10] M. Qiu and Y. Song, "Predicting the Direction of Stock Market Index Movement Using an Optimized Artificial Neural Network Model", PLOS ONE, Vol. 11, No. 5, 2016.

[11] E. Berner, Clinical decision support systems, 2nd ed., Springer, Ed. New York, NY: Springer Science and Business Media, LLC, 2007.

[12] X. Yong, W. Hongwei, and F. Qi, "Electronic market models for decision support systems on the Web", Journal of Systems Engineering and Electronics, Vol. 15, No. 2, pp. 135-141, 2004.
[13] S. Huang, Y. Hung, and D. Yen, "A study on decision factors in adopting an online stock trading system by brokers in Taiwan", Decision Support Systems, Vol. 40, No. 2, pp. 315-328, 2005.

[14] P. Chatzoglou and K. Chourmouziadis, "An intelligent short term stock trading fuzzy system for assisting investors in portfolio management", Expert Systems with Applications, Vol. 43, pp. 298-311, 2016.

[15] G. Box, G. Jenkins, G. Reinsel, and G. Ljung, Time series analysis: Forecasting and control, 3rd ed.: Hoboken, NJ: John Wiley \& Sons, 2013.

[16] L. Zadeh, "Fuzzy Sets", Information and Control, Vol. 8, pp. 338-353, 1965.

[17] B. Nair, M. Minuvarthini, B. Sujithra, and V. Mohandas, "Stock Market Prediction Using a Hybrid Neuro-fuzzy System", In: Proc. of International Conf. On Advances in Recent Technologies in Communication and Computing, pp. 243 -247, 2010.

[18] L. Maciel, F. Gomide, and R. Ballini, "Stock market volatility prediction using possibilistic fuzzy modeling", In: Proc. of International Conf. on Computational Intelligence, LA, 2015.

[19] S. Bharathi and A. Geetha, "Sentiment Analysis for Effective Stock Market Prediction", International Journal of Intelligent Engineering and Systems, Vol. 10, No. 3, pp. 146-154, 2017.

[20] H. Kwaśnicka and M. Ciosmak, "Intelligent Techniques in Stock Analysis", Intelligent Information Systems, Vol. 10, pp. 195-208, 2001.

[21] R. Vince, Portfolio management formulas, New York: Willey \& Sons, 1990.

[22] C. Lin, "A fuzzy decision support system for strategic portfolio management", Decision Support Systems, Vol. 38, No. 3, pp. 383 - 398, 2004. 